REVISTA DE LA

UNIÓN MATEMÁTICA ARGENTINA

Vol. 59, No. 2, 2018, Pages 285-309

Published online: February 23, 2018

\title{
A REDUCTION FORMULA FOR LENGTH-TWO POLYLOGARITHMS AND SOME APPLICATIONS
}

\author{
MATILDE N. LALÍN AND JEAN-SÉBASTIEN LECHASSEUR
}

\begin{abstract}
We use shuffle and stuffle relations to give a simple proof of a reduction formula for length-two multiple polylogarithms evaluated in complex parameters of absolute value 1 in terms of a finite sum of products of lengthone polylogarithms. This result was originally due to Nakamura and recently reproved by Panzer by different methods. This generalises results of Borwein and Girgensohn for alternating Euler sums and for multiple zeta values twisted by fourth roots of unity by the first author. We also explore implications for other colored multiple zeta values and present some applications to Mahler measure and Feynman diagrams.
\end{abstract}

\section{INTRODUCTION}

The study of multiple zeta values and their generalisations, multiple polylogarithms, has been attracting great interest for several years. For $n_{1}, \ldots, n_{m}$ positive integers, one can define the multiple polylogarithm as the complex function given by the power series

$$
\operatorname{Li}_{n_{1}, \ldots, n_{m}}\left(w_{1}, \ldots, w_{m}\right):=\sum_{0<j_{1}<\cdots<j_{m}} \frac{w_{1}^{j_{1}} \cdots w_{m}^{j_{m}}}{j_{1}^{n_{1}} \cdots j_{m}^{n_{m}}},
$$

which is absolutely convergent for $\left|w_{i}\right| \leq 1$ for $i=1, \ldots, m-1$ and $\left|w_{m}\right| \leq 1$ (respectively $\left|w_{m}\right|<1$ ) if $n_{m}>1$ (resp. $n_{m}=1$ ). This function can also be extended to a multi-valued meromorphic function on $\mathbb{C}^{m}$. The number $m$ is called the length (or depth) of the polylogarithm, and the number $n_{1}+\cdots+n_{m}$ is called the weight.

2010 Mathematics Subject Classification. Primary 11G55; Secondary 11M06, 11R06.

Key words and phrases. colored multiple zeta values, Euler sums, cyclotomic multiple polylogarithms, alternating multiple zeta values, special values of $\zeta(s)$ and Dirichlet $L$-functions, double Lerch values.

This work was supported by the Natural Sciences and Engineering Research Council of Canada (Discovery Grant 355412-2013) and the Fonds de recherche du Québec - Nature et technologies (Établissement de nouveaux chercheurs 144987 to ML, Bourse de doctorat en recherche (B2) 175957 to JSL). 
For example, when $m=1$ and $n_{1}=1$, we obtain the Taylor series of the logarithm around 1 ,

$$
\operatorname{Li}_{1}(w)=\sum_{j=1}^{\infty} \frac{w^{j}}{j}=-\log (1-w) .
$$

Special values of multiple polylogarithms when $w_{i}=1$ for $i=1, \ldots, m$ are called multiple zeta values. Indeed, the simplest possible example yields the Riemann zeta function

$$
\operatorname{Li}_{n}(1)=\sum_{j=1}^{\infty} \frac{1}{j^{n}}=\zeta(n) .
$$

Multiple zeta values have been studied in different contexts. We refer the reader to the excellent website by Hoffman [14] with a compilation of references in the subject.

When the $w_{i}$ are chosen to be roots of unity of order $N$, one obtains multiple zeta values twisted by roots of unity (also called colored multiple zeta values, Euler sums, or Zagier sums). We will say that such values have order $N$ to indicate the order of the roots of unity involved. Much like ordinary multiple zeta values, colored multiple zeta values are also often related to special values of functions of number theoretic interest. For instance, taking $m=1$ and $w_{1}=-1$, we get the Riemann zeta function again,

$$
\operatorname{Li}_{n}(-1)=\left(2^{1-n}-1\right) \zeta(n) .
$$

Taking $w= \pm i$, we obtain a relationship with a Dirichlet $L$-function

$$
\begin{aligned}
\operatorname{Im}\left(\operatorname{Li}_{n}(i)\right)=\frac{\operatorname{Li}_{n}(i)-\operatorname{Li}_{n}(-i)}{2 i} & =\frac{1}{2 i} \sum_{j=1}^{\infty} \frac{i^{j}\left(1-(-1)^{j}\right)}{j^{n}} \\
& =\frac{1}{2 i} \sum_{j \text { odd }} \frac{2 i^{j}}{j^{n}}=\sum_{j=0}^{\infty} \frac{(-1)^{j}}{(2 j+1)^{n}} \\
& =L\left(\chi_{-4}, n\right) .
\end{aligned}
$$

Here $L$ denotes the Dirichlet $L$-function in the character of conductor 4 , given by

$$
L\left(\chi_{-4}, s\right):=\sum_{j=1}^{\infty} \frac{\chi_{-4}(j)}{j^{s}}, \quad \chi_{-4}(j)=\left\{\begin{array}{cl}
1 & j \equiv 1 \bmod 4 \\
-1 & j \equiv-1 \bmod 4 \\
0 & j \text { even } .
\end{array}\right.
$$

Similarly, one can see that

$$
\operatorname{Re}\left(\operatorname{Li}_{n}(i)\right)=\frac{\operatorname{Li}_{n}(i)+\operatorname{Li}_{n}(-i)}{2}=\left(2^{1-2 n}-2^{-n}\right) \zeta(n) .
$$

Generally speaking there are known functional equations among multiple polylogarithms (for instance, shuffle and stuffle relations) which yield further relationships among colored multiple zeta values. A major problem consists of finding the dimension of the $\mathbb{Q}$-vector space spanned by colored multiple zeta values of a given weight and order. This has recently been established for multiple zeta values by Brown [8] and Zagier [26] from an original conjecture of Hoffman [13]. 
Euler [10] was the first to study the case $m=2$ and showed the following result.

Theorem 1. For positive integers $r, s$ such that $k=r+s$ is odd and $r$ is even,

$$
\zeta(r, s)=-\frac{1}{2}\left(\left(\begin{array}{l}
k \\
r
\end{array}\right)+1\right) \zeta(k)+\sum_{\substack{a+b=k \\
a, b \geq 2 \\
a \text { odd }}}\left(\left(\begin{array}{l}
a-1 \\
r-1
\end{array}\right)+\left(\begin{array}{l}
a-1 \\
s-1
\end{array}\right)\right) \zeta(a) \zeta(b),
$$

where $\zeta(r, s)=\operatorname{Li}_{r, s}(1,1)$.

Thus, Euler's result shows that some multiple polylogarithms of length two evaluated at $w_{1}=w_{2}=1$ can be written in terms of sums of products of polylogarithms of length one.

The following generalisation of Theorem 1 is due to Borwein and Girgensohn (see [3, eq. (75)] and [4] for more details).

Theorem 2. For $\rho, \sigma= \pm 1$ and $r, s$ positive integers such that $k=r+s$ is odd, we have

$$
\begin{aligned}
\operatorname{Li}_{r, s}(\rho, \sigma)= & \frac{1}{2}\left(-\operatorname{Li}_{k}(\rho \sigma)+\left(1+(-1)^{s}\right) \operatorname{Li}_{r}(\rho) \operatorname{Li}_{s}(\sigma)\right) \\
& +\frac{(-1)^{s}}{2}\left(\left(\begin{array}{c}
k-1 \\
r-1
\end{array}\right) \operatorname{Li}_{k}(\rho)+\left(\begin{array}{c}
k-1 \\
s-1
\end{array}\right) \operatorname{Li}_{k}(\sigma)\right) \\
& -(-1)^{s} \sum_{\substack{m=1 \\
m \text { odd }}}^{k-1}\left(\left(\begin{array}{c}
m-1 \\
r-1
\end{array}\right) \operatorname{Li}_{m}(\rho)+\left(\begin{array}{c}
m-1 \\
s-1
\end{array}\right) \operatorname{Li}_{m}(\sigma)\right) \operatorname{Li}_{k-m}(\rho \sigma) .
\end{aligned}
$$

There are several directions one can explore to generalise this result further. The most obvious ones would be to try larger lengths or orders higher than 2 .

In this note, we will treat the higher order case. More precisely, our first goal is to provide a new proof to the following result.

Theorem 3. Let $r, s$ be positive integers, $k=r+s$, and let $u, v$ be complex numbers such that $|u|=|v|=1$. In addition, we assume that $v \neq 1$ if $s=1$. Let

$$
\operatorname{Re}_{k}= \begin{cases}\operatorname{Re} & k \text { odd } \\ i \operatorname{Im} & k \text { even }\end{cases}
$$

Then,

$$
\begin{aligned}
2 \operatorname{Re}_{k}\left(\operatorname{Li}_{r, s}(u, v)\right)= & (-1)^{k} \operatorname{Li}_{k}(\overline{u v})+(-1)^{k+1} \operatorname{Li}_{r}(\bar{u}) \operatorname{Li}_{s}(\bar{v})+(-1)^{r-1} \operatorname{Li}_{r}(\bar{u}) \operatorname{Li}_{s}(v) \\
+ & (-1)^{r-1}\left(\left(\begin{array}{c}
k-1 \\
r-1
\end{array}\right) \operatorname{Li}_{k}(\bar{u})+\left(\begin{array}{c}
k-1 \\
s-1
\end{array}\right) \operatorname{Li}_{k}(v)\right) \\
& +\sum_{m=1}^{k-1}\left(\left(\begin{array}{c}
m-1 \\
r-1
\end{array}\right) \operatorname{Li}_{m}(\bar{u})+\left(\begin{array}{c}
m-1 \\
s-1
\end{array}\right)(-1)^{k+m} \operatorname{Li}_{m}(v)\right) \\
& \times\left((-1)^{r} \operatorname{Li}_{k-m}(u v)+(-1)^{s+m} \operatorname{Li}_{k-m}(\overline{u v})\right) .
\end{aligned}
$$

In the formula above as well as in the formula of Theorem 2 we may occasionally have nonconvergent terms such as $\operatorname{Li}_{1}(1)$. When this is the case, nonconvergent 
terms cancel and the formulas are to be interpreted by ignoring such terms (or assigning them an arbitrary value by the process known as regularisation).

Theorem 3 was first proved by Nakamura as Proposition 2.1 in $[22]^{1}$ The result was deduced from a more general statement involving a functional relation of a Lerch type Tornheim double zeta function. Nakamura gives a very elegant proof using elementary combinatorial facts and the analytic continuation of the Torheim series. A more general result (without restricting to $|u|=|v|=1$ ) was recently recovered again by Panzer as equation (3.2) in [24] in a more general setting. Panzer formulated and proved a beautiful parity theorem for multiple polylogarithms. The parity theorem predicts that under certain parity conditions on the weight and length, a multiple zeta value will reduce to combinations of lower length multiple zeta values. Panzer used differentiation and shuffle relations to prove some reductions of polylogarithms to certain evaluations of Bernoulli polynomials and then found explicit reductions for lengths 2 and 3 .

For our proof, we use the generating polynomials of the shuffle and stuffle relations for polylogarithms of a fixed weight. We were inspired by the ideas from [5], p. 44] where they consider the multiple zeta case.

It is immediate to see Theorem 2 as a consequence of Theorem 3 We may specialize in other roots of unity in order to get other corollaries.

We remark that in the formula above we only need that $u, v$ are complex numbers of absolute value one, not necessarily roots of unity. Such values are known as double Lerch values and were already studied by Arakawa and Kaneko [2] and Nakamura [20, 21].

Our motivation for highlighting Theorem 3 is that it finds several applications in different areas that require lowering the length of polylogarithms. Thus, the second goal of this article consists on exploring consequences of Theorem 3 , particularly in the realm of Mahler measure.

Given a nonzero multivariable rational function $P \in \mathbb{C}\left(x_{1}, \ldots, x_{n}\right)$, its (logarithmic) Mahler measure is defined by

$$
\mathrm{m}(P):=\frac{1}{(2 \pi i)^{n}} \int_{\mathbb{T}^{n}} \log \left|P\left(x_{1}, \ldots, x_{n}\right)\right| \frac{\mathrm{d} x_{1}}{x_{1}} \cdots \frac{\mathrm{d} x_{n}}{x_{n}},
$$

where $\mathbb{T}^{n}=\left\{\left(z_{1}, \ldots, z_{n}\right) \in \mathbb{C}^{n}|| z_{1}|=\cdots=| z_{n} \mid=1\right\}$.

This construction was originally considered in the context of searching for large prime numbers and of classical heights of polynomials. Later it was found to yield special values of the Riemann zeta function and $L$-functions of number theoretic interest as well as values of polylogarithms and other special functions. These phenomena have been partially explained in terms of Beilinson's conjectures via relationships with regulators by Deninger [9] (see also the works of Boyd [6] and Rodriguez-Villegas 25] for more insights on these aspects). In this setting it is easier to understand special values of polylogarithms of length 1 than those of higher length in terms of the regulator. Therefore, it is desirable to obtain formulas for Mahler measure and higher Mahler measure where the value is expressed in terms

\footnotetext{
${ }^{1}$ The statement of Proposition 2.1 in 22 has a minor typo which we correct here in Theorem 3
} 
of length 1 polylogarithms. It is in this context that Theorem 3 finds an important application. For example we can write

$$
\begin{aligned}
\mathrm{m} & \left(1+x+\left(\frac{1-x_{1}}{1+x_{1}}\right) \cdots\left(\frac{1-x_{2 n+1}}{1+x_{2 n+1}}\right)(1+y) z\right) \\
= & \sum_{h=0}^{n} \frac{s_{n-h}\left(1^{2}, 3^{2}, \ldots,(2 n-1)^{2}\right)}{(2 n) !}\left(\frac{2}{\pi}\right)^{2 h+3} \\
& \left.\times \sum_{\ell=0}^{h}\left(\begin{array}{c}
2 h+1 \\
2 \ell
\end{array}\right) \frac{(2 h+3-2 \ell) !}{2(2 h+1)}(-1)^{\ell} B_{2 \ell} \pi^{2 \ell} L\left(\chi_{-4}, 2 h+4-2 \ell\right)\right),
\end{aligned}
$$

where the $s_{l}\left(a_{1}, \ldots, a_{k}\right)$ coefficients are given by

$$
s_{l}\left(a_{1}, \ldots, a_{k}\right)= \begin{cases}1 & \text { if } l=0, \\ \sum_{i_{1}<\cdots<i_{l}} a_{i_{1}} \cdots a_{i_{l}} & \text { if } 0<l \leq k, \\ 0 & \text { if } k<l\end{cases}
$$

The Mahler measure of $1+x+\left(\frac{1-x_{1}}{1+x_{1}}\right) \cdots\left(\frac{1-x_{2 n+1}}{1+x_{2 n+1}}\right)(1+y) z$ was first computed in [16, but the formula given there involves polylogarithms of length 2 evaluated at fourth roots of unity. Theorem 3 allows us to write formula (1) solely in terms of Dirichlet $L$-functions. The new formulation was announced in [19] without proof. We provide here the details leading to this formula.

Theorem 3 has applications beyond Mahler measure. As another example, we present a simplification of some of the sums

$$
V_{s, r}=\sum_{0<j<\ell} \frac{(-1)^{\ell} \cos (2 \pi j / 3)}{j^{r} \ell^{s}}
$$

in terms of the Riemann zeta-function and Dirichlet $L$-functions. This type of sum appears in connection with Feynman diagrams [7].

This paper is organised as follows. In Section 2 we state the definitions of polylogarithms, hyperlogarithms and their relevant properties such as the shuffle and stuffle relations. We prove our result in Section 3 We discuss some particular cases (including Feynman diagrams) in Section 4 and focus on applications to Mahler measure in Section 5 Finally, we discuss further directions in Section 6. We intend for this paper to be relatively self contained. To this end, we have included several facts that are well-known to the experts.

\section{Polylogarithms and hyperlogarithms}

Let $w_{1}, \ldots, w_{m}$ be complex variables and $n_{1}, \ldots, n_{m}$ be positive integers. Recall from the introduction that we defined the multiple polylogarithm as

$$
\operatorname{Li}_{n_{1} \cdots n_{m}}\left(w_{1}, \ldots, w_{m}\right):=\sum_{0<j_{1}<\cdots<j_{m}} \frac{w_{1}^{j_{1}} \cdots w_{m}^{j_{m}}}{j_{1}^{n_{1}} \cdots j_{m}^{n_{m}}} .
$$


As we mentioned before, the series above is absolutely convergent for $\left|w_{i}\right| \leq 1$ for $i=1, \ldots, m-1$ and $\left|w_{m}\right| \leq 1$ (respectively $\left|w_{m}\right|<1$ ) if $n_{m}>1$ (resp. $n_{m}=1$ ). Moreover, for $n_{m}=1$, the series converges as long as $w_{m} \neq 1$.

Multiple polylogarithms have meromorphic continuation to the complex plane. The hyperlogarithm is defined by the following iterated integral:

$$
\begin{aligned}
& \mathrm{I}_{n_{1} \cdots n_{m}}\left(a_{1}: \cdots: a_{m+1}\right) \\
& :=\int_{0}^{a_{m+1}} \underbrace{\frac{d t}{t-a_{1}} \circ \frac{d t}{t} \circ \cdots \circ \frac{d t}{t}}_{n_{1}} \circ \underbrace{\frac{d t}{t-a_{2}} \circ \frac{d t}{t} \circ \cdots \circ \frac{d t}{t}}_{n_{2}} \\
& \circ \cdots \circ \underbrace{\frac{d t}{t-a_{m}} \circ \frac{d t}{t} \circ \cdots \circ \frac{d t}{t}}_{n_{m}},
\end{aligned}
$$

where

$$
\int_{0}^{b_{h+1}} \frac{d t}{t-b_{1}} \circ \cdots \circ \frac{d t}{t-b_{h}}=\int_{0 \leq t_{1} \leq \cdots \leq t_{h} \leq b_{h+1}} \frac{d t_{1}}{t_{1}-b_{1}} \cdots \frac{d t_{h}}{t_{h}-b_{h}}
$$

The path of integration should be interpreted as any path connecting 0 and $b_{h+1}$ in $\mathbb{C} \backslash\left\{b_{1}, \ldots, b_{h}\right\}$. The integral depends on the homotopy class of this path.

Multiple polylogarithms and hyperlogarithms are related by the following identities (see [11]):

$$
\begin{aligned}
\mathrm{I}_{n_{1} \cdots n_{m}}\left(a_{1}: \cdots: a_{m+1}\right) & =(-1)^{m} \operatorname{Li}_{n_{1} \cdots n_{m}}\left(\frac{a_{2}}{a_{1}}, \frac{a_{3}}{a_{2}}, \cdots, \frac{a_{m+1}}{a_{m}}\right), \\
\operatorname{Li}_{n_{1} \cdots n_{m}}\left(w_{1}, \ldots, w_{m}\right) & =(-1)^{m} \mathrm{I}_{n_{1} \cdots n_{m}}\left(\left(w_{1} \cdots w_{m}\right)^{-1}: \cdots: w_{m}^{-1}: 1\right) .
\end{aligned}
$$

Polylogarithms satisfy the shuffle and stuffle relations. Roughly speaking, the stuffle product is the result of multiplying two or more multiple polylogarithms when they are expressed in terms of series, while the shuffle product is the result of doing this multiplication at the level of the integrals, or more precisely, hyperlogarithms. For the purpose of this paper we only need to multiply two polylogarithms of length one, and therefore we will only describe these two cases.

When the corresponding power series converge, the stuffle product of $\operatorname{Li}_{r}(u)$ and $\mathrm{Li}_{s}(v)$ is constructed from the power series as follows:

$$
\begin{aligned}
\operatorname{Li}_{r}(u) * \operatorname{Li}_{s}(v) & :=\left(\sum_{j=1}^{\infty} \frac{u^{j}}{j^{r}}\right)\left(\sum_{\ell=1}^{\infty} \frac{v^{\ell}}{\ell^{s}}\right) \\
& =\sum_{0<j<\ell} \frac{u^{j} v^{\ell}}{j^{r} \ell^{s}}+\sum_{0<j<\ell} \frac{v^{j} u^{\ell}}{j^{s} \ell^{r}}+\sum_{j=1}^{\infty} \frac{(u v)^{j}}{j^{r+s}} \\
& =\operatorname{Li}_{r, s}(u, v)+\operatorname{Li}_{s, r}(v, u)+\operatorname{Li}_{r+s}(u v) .
\end{aligned}
$$


The shuffle product of $\operatorname{Li}_{r}(u)$ and $\operatorname{Li}_{s}(v)$ is constructed from the corresponding hyperlogarithm integrals as follows:

$$
\begin{aligned}
& \operatorname{Li}_{r}(u) m \operatorname{Li}_{s}(v):=(-\int_{0}^{1} \underbrace{\frac{d t}{t-u^{-1}} \circ \frac{d t}{t} \circ \cdots \circ \frac{d t}{t}}_{r})(-\int_{0}^{1} \underbrace{\frac{d t}{t-v^{-1}} \circ \frac{d t}{t} \circ \cdots \circ \frac{d t}{t}}_{s}) \\
& =\sum_{h=0}^{r-1}\left(\begin{array}{c}
s-1+h \\
s-1
\end{array}\right) \int_{0}^{1} \underbrace{\frac{d t}{t-u^{-1}} \circ \frac{d t}{t} \circ \cdots \circ \frac{d t}{t}}_{r-h} \circ \underbrace{\frac{d t}{t-v^{-1}} \circ \frac{d t}{t} \circ \cdots \circ \frac{d t}{t}}_{s+h} \\
& +\sum_{h=0}^{s-1}\left(\begin{array}{c}
r-1+h \\
r-1
\end{array}\right) \int_{0}^{1} \underbrace{\frac{d t}{t-v^{-1}} \circ \frac{d t}{t} \circ \cdots \circ \frac{d t}{t}}_{s-h} \circ \underbrace{\frac{d t}{t-u^{-1}} \circ \frac{d t}{t} \circ \cdots \circ \frac{d t}{t}}_{r+h} \\
& =\sum_{h=0}^{r-1}\left(\begin{array}{c}
s-1+h \\
s-1
\end{array}\right) I_{r-h, s+h}\left(u^{-1}: v^{-1}: 1\right)+\sum_{h=0}^{s-1}\left(\begin{array}{c}
r-1+h \\
r-1
\end{array}\right) I_{s-h, r+h}\left(v^{-1}: u^{-1}: 1\right) \\
& =\sum_{h=0}^{r-1}\left(\begin{array}{c}
s-1+h \\
s-1
\end{array}\right) \operatorname{Li}_{r-h, s+h}\left(u v^{-1}, v\right)+\sum_{h=0}^{s-1}\left(\begin{array}{c}
r-1+h \\
r-1
\end{array}\right) \operatorname{Li}_{s-h, r+h}\left(u^{-1} v, u\right) \\
& =\sum_{m=1}^{r+s-1}\left[\left(\begin{array}{c}
m-1 \\
s-1
\end{array}\right) \operatorname{Li}_{r+s-m, m}\left(u v^{-1}, v\right)+\left(\begin{array}{c}
m-1 \\
r-1
\end{array}\right) \operatorname{Li}_{r+s-m, m}\left(u^{-1} v, u\right)\right] \text {. }
\end{aligned}
$$

At the same time, we remark that $\operatorname{Li}_{r}(u) * \operatorname{Li}_{s}(v)$ and $\operatorname{Li}_{r}(u) \amalg \operatorname{Li}_{s}(v)$ are both simply equal to $\operatorname{Li}_{r}(u) \operatorname{Li}_{s}(v)$.

\section{THE MAIN RESULT}

The goal of this section is to prove Theorem 3 . For this, we will adapt the ideas from [5, p. 44] to the more general case of polylogarithms. The strategy for the proof is to combine all the shuffle and stuffle relations together for a fixed total weight $k$. In order to do this we need to be able to take any positive value for $r$ and $s$.

From now on, we work under the conditions of Theorem 3 In particular we assume that $|u|=|v|=1$, which allows us to write $u^{-1}=\bar{u}$ and $v^{-1}=\bar{v}$.

Now observe that all the series involved in (2) and (3) converge as long as $r, s>1$ or $u \neq 1$ (respectively $v \neq 1$ ) for $r=1$ (resp. $s=1$ ). Theorem 3 already has the condition that $v \neq 1$ for $s=1$. Before proceeding with the proof of Theorem 3 we need to clarify what happens when $u=1$ and $r=1$. If we were to write equations (2) and (3) with $r=1, u=1$, we would get the following:

$$
\begin{aligned}
& \operatorname{Li}_{1}(1) * \operatorname{Li}_{s}(v)=\operatorname{Li}_{1, s}(1, v)+\operatorname{Li}_{s, 1}(v, 1)+\operatorname{Li}_{1+s}(v), \\
& \operatorname{Li}_{1}(1) \amalg \operatorname{Li}_{s}(v)=\operatorname{Li}_{1, s}(\bar{v}, v)+\operatorname{Li}_{s, 1}(v, 1)+\sum_{m=2}^{s} \operatorname{Li}_{s+1-m, m}(v, 1) .
\end{aligned}
$$


It should be noted that the equations above have no mathematical meaning because neither of the terms $\operatorname{Li}_{1}(1)$ nor $\operatorname{Li}_{s, 1}(v, 1)$ converge. We claim that we can give any arbitrary value to $\mathrm{Li}_{1}(1)$ and let that define the value of $\mathrm{Li}_{s, 1}(v, 1)$ by means of any of the above equations. This process is called regularisation and it depends on the consistency of the two equations above. In order for this reasoning to work, we need to prove the following result.

Proposition 4. Let $s$ be a positive integer and $v$ a complex number with $|v|=1$. Further assume that $v \neq 1$ if $s=1$. Then

$$
\sum_{m=2}^{s} \operatorname{Li}_{s+1-m, m}(v, 1)=\operatorname{Li}_{1, s}(1, v)+\operatorname{Li}_{1+s}(v)-\operatorname{Li}_{1, s}(\bar{v}, v) .
$$

We remark that this equation is a polylogarithmic version of a particular case of the Sum Theorem due to Granville ([12, Proposition, p. 1) and Zagier:

$$
\zeta(k)=\sum_{\substack{s_{1}+\ldots+s_{d}=k \\ s_{i} \geq 1, s_{d} \geq 2}} \zeta\left(s_{1}, \ldots, s_{d}\right)
$$

Proof. We will adapt Granville's idea to our case. We start by developing

$$
\sum_{m=2}^{s} \operatorname{Li}_{s+1-m, m}(v, 1)=\sum_{0<j<\ell} v^{j} \sum_{\substack{m+n=s+1 \\ 1<m, 0<n}} \frac{1}{j^{n} \ell^{m}} .
$$

The inner sum in the right-hand side is the coefficient of $x^{s+1}$ in the power series

$$
\begin{aligned}
\left(\sum_{1<m}\left(\frac{x}{\ell}\right)^{m}\right)\left(\sum_{0<n}\left(\frac{x}{j}\right)^{n}\right) & =\left(\frac{x^{2} / \ell^{2}}{1-x / \ell}\right)\left(\frac{x / j}{1-x / j}\right) \\
& =\frac{x^{3} / \ell}{(j-x)(\ell-x)} \\
& =\frac{x^{3}}{\ell(\ell-j)}\left(\frac{1}{j-x}-\frac{1}{\ell-x}\right) .
\end{aligned}
$$

Thus the sum in the right-hand side of (4) becomes

$$
\sum_{0<j<\ell} \frac{v^{j}}{\ell(\ell-j)}\left(\frac{1}{j^{s-1}}-\frac{1}{\ell^{s-1}}\right)=\sum_{0<j<\ell} \frac{v^{j}}{\ell(\ell-j) j^{s-1}}-\sum_{0<j<\ell} \frac{v^{j}}{\ell^{s}(\ell-j)} .
$$

Notice that

$$
\sum_{0<j<\ell} \frac{v^{j}}{\ell(\ell-j) j^{s-1}}=\sum_{0<j, h} \frac{v^{j}}{j^{s-1} h(h+j)}
$$

and

$$
\sum_{0<h} \frac{1}{h(h+j)}=\frac{1}{j} \sum_{0<h}\left(\frac{1}{h}-\frac{1}{h+j}\right)=\frac{1}{j} \sum_{h=1}^{j} \frac{1}{h}=\frac{1}{j^{2}}+\sum_{h=1}^{j-1} \frac{1}{h j} .
$$


By replacing in (6) and (5), we obtain

$$
\begin{aligned}
\sum_{m=2}^{s} \operatorname{Li}_{s+1-m, m}(v, 1) & =\sum_{0<j} \frac{v^{j}}{j^{s+1}}+\sum_{0<h<j} \frac{v^{j}}{h j^{s}}-\sum_{0<\ell-j<\ell} \frac{\bar{v}^{\ell-j} v^{\ell}}{(\ell-j) \ell^{s}} \\
& =\operatorname{Li}_{s+1}(v)+\operatorname{Li}_{1, s}(1, v)-\operatorname{Li}_{1, s}(\bar{v}, v) .
\end{aligned}
$$

This concludes the proof of Proposition 4.

Because of Proposition 4, we can assign any value to $\mathrm{Li}_{1}(1)$ and deduce from there a consistent value for $\operatorname{Li}_{s, 1}(v, 1)$. The same is true for $\operatorname{Li}_{r, 1}(u, 1)$. But we must still exclude the case of $\operatorname{Li}_{1,1}(1,1)$, as this term can not be defined consistently with the shuffle and stuffle relations. For the purpose of Theorem 3 , this case is already excluded for the conditions that we impose on $s$ and $v$.

We are now ready to prove our main result.

Proof of Theorem 3. We fix $k$ and we consider the following two families of twovariable polynomials that are indexed by all the possible pairs $\{u, v\}$ :

$$
\begin{aligned}
& P_{u, v}(x, y)=\sum_{\substack{r+s=k \\
r, s \geq 1}} \operatorname{Li}_{r}(u) \operatorname{Li}_{s}(v) x^{r-1} y^{s-1}, \\
& Q_{u, v}(x, y)=\sum_{\substack{m+n=k \\
m, n \geq 1}} \operatorname{Li}_{m, n}(u, v) x^{m-1} y^{n-1} .
\end{aligned}
$$

The first family is symmetric with respect to exchanging the variables and the indexes at the same time.

$$
P_{u, v}(x, y)=P_{v, u}(y, x) .
$$

It will be convenient to define the complex conjugate for a polynomial $T(x, y)=$ $\sum a_{j, \ell} x^{j} y^{\ell} \in \mathbb{C}[x, y]$ as conjugating each coefficient in the following way: $\overline{T(x, y)}=$ $\sum \frac{a_{j, \ell}}{a^{j}} y^{\ell}$. The real and imaginary parts of $T(x, y)$ will be defined similarly.

With this notation in mind, we have

$$
P_{\bar{u}, \bar{v}}(x, y)=\overline{P_{u, v}(x, y)}, \quad Q_{\bar{u}, \bar{v}}(x, y)=\overline{Q_{u, v}(x, y)} .
$$

Changing the signs of both variables at the same time allows us to capture the parity of the weight $k$ in both polynomial families.

$$
P_{u, v}(-x,-y)=(-1)^{k} P_{u, v}(x, y), \quad Q_{u, v}(-x,-y)=(-1)^{k} Q_{u, v}(x, y) .
$$

After recording all the basic properties of $P_{u, v}(x, y)$ and $Q_{u, v}(x, y)$, we proceed to use these two polynomial families to express the stuffle and shuffle relations. The stuffle relations (2) become

$$
P_{u, v}(x, y)=Q_{u, v}(x, y)+Q_{v, u}(y, x)+\operatorname{Li}_{k}(u v) \frac{x^{k-1}-y^{k-1}}{x-y} .
$$

The shuffle relations (3) become

$$
P_{u, v}(x, y)=Q_{u \bar{v}, v}(x, x+y)+Q_{v \bar{u}, u}(y, x+y) .
$$


From (11) we have the following:

$$
\begin{aligned}
P_{u v, v}(x, y-x) & =Q_{u, v}(x, y)+Q_{\bar{u}, u v}(y-x, y), \\
P_{v, \bar{u}}(y,-x) & =Q_{u v, \bar{u}}(y, y-x)+Q_{\overline{u v}, v}(-x, y-x), \\
P_{\bar{u}, \overline{u v}}(y-x,-y) & =Q_{v, \overline{u v}}(y-x,-x)+Q_{\bar{v}, \bar{u}}(-y,-x) .
\end{aligned}
$$

Adding the three equations above, we obtain

$$
\begin{aligned}
P_{u v, v}(x, y-x)+P_{v, \bar{u}}(y,-x)+P_{\bar{u}, \overline{u v}}(y-x,-y) \\
=Q_{u, v}(x, y)+\left(Q_{\bar{u}, u v}(y-x, y)+Q_{u v, \bar{u}}(y, y-x)\right) \\
\quad+\left(Q_{\overline{u v}, v}(-x, y-x)+Q_{v, \overline{u v}}(y-x,-x)\right)+Q_{\bar{v}, \bar{u}}(-y,-x) .
\end{aligned}
$$

Now we use 10 inside the above parentheses and for $P_{\bar{u}, \bar{v}}(-x,-y)$ in order to replace the term $Q_{\bar{v}, \bar{u}}(-y,-x)$ in the right-hand side above.

$$
\begin{aligned}
P_{u v, v}(x, y-x)+P_{v, \bar{u}}(y,-x)+P_{\bar{u}, \overline{u v}}(y-x,-y) \\
=Q_{u, v}(x, y)+\left(P_{\bar{u}, u v}(y-x, y)+\operatorname{Li}_{k}(v) \frac{(y-x)^{k-1}-y^{k-1}}{x}\right) \\
\quad+\left(P_{\overline{u v}, v}(-x, y-x)+\operatorname{Li}_{k}(\bar{u}) \frac{(-x)^{k-1}-(y-x)^{k-1}}{y}\right) \\
\quad+\left(P_{\bar{u}, \bar{v}}(-x,-y)-Q_{\bar{u}, \bar{v}}(-x,-y)+\operatorname{Li}_{k}(\overline{u v}) \frac{(-x)^{k-1}-(-y)^{k-1}}{x-y}\right) .
\end{aligned}
$$

Rearranging and using the symmetric relation (7), this yields

$$
\begin{aligned}
& Q_{u, v}(x, y)-Q_{\bar{u}, \bar{v}}(-x,-y) \\
& =P_{\bar{u}, v}(-x, y)+P_{\bar{u}, \overline{u v}}(y-x,-y)+P_{v, u v}(y-x, x) \\
& \quad-\left(P_{\bar{u}, \bar{v}}(-x,-y)+P_{\bar{u}, u v}(y-x, y)+P_{v, \overline{u v}}(y-x,-x)\right) \\
& \quad-\left(\operatorname{Li}_{k}(\overline{u v}) \frac{(-x)^{k-1}-(-y)^{k-1}}{x-y}+\operatorname{Li}_{k}(v) \frac{(y-x)^{k-1}-y^{k-1}}{x}\right. \\
& \left.\quad+\operatorname{Li}_{k}(\bar{u}) \frac{(-x)^{k-1}-(y-x)^{k-1}}{y}\right) .
\end{aligned}
$$

Using (8) and (9),

$$
\begin{aligned}
Q_{u, v}(x, y)+ & (-1)^{k+1} \overline{Q_{u, v}(x, y)} \\
=P_{\bar{u}, v}(-x, y)+ & P_{\bar{u}, \overline{u v}}(y-x,-y)+P_{v, u v}(y-x, x) \\
& +(-1)^{k+1}\left(P_{\bar{u}, \bar{v}}(x, y)+P_{\bar{u}, u v}(x-y,-y)+P_{v, \overline{u v}}(x-y, x)\right) \\
& +(-1)^{k}\left(\operatorname{Li}_{k}(\overline{u v}) \frac{x^{k-1}-y^{k-1}}{x-y}+\operatorname{Li}_{k}(v) \frac{(x-y)^{k-1}-(-y)^{k-1}}{x}\right. \\
& \left.+\operatorname{Li}_{k}(\bar{u}) \frac{(x-y)^{k-1}-x^{k-1}}{-y}\right) .
\end{aligned}
$$


Recall from the statement of Theorem 3 that we defined $\mathrm{Re}_{k}$ to be equal to Re for $k$ odd and to $i \mathrm{Im}$ for $k$ even. Then we deduce:

$$
2 \operatorname{Re}_{k}\left(Q_{u, v}(x, y)\right)=R_{\bar{u}, \bar{v}}(x, y)+R_{\bar{u}, u v}(x-y,-y)+R_{v, \overline{u v}}(x-y, x)
$$

where

$$
R_{u, v}(x, y)=(-1)^{k+1} P_{u, v}(x, y)+P_{u, \bar{v}}(-x, y)+(-1)^{k} \operatorname{Li}_{k}(u v) \frac{x^{k-1}-y^{k-1}}{x-y} .
$$

We now fix the pair $r, s$ such that $r+s=k$. We have to determine the coefficient of $x^{r-1} y^{s-1}$ in each of the terms in the right-hand side of 12 . Let $[\cdot]_{a, b}$ denote the coefficient of $x^{a} y^{b}$. Thus,

$$
\begin{aligned}
& {\left[R_{\bar{u}, \bar{v}}(x, y)\right]_{r-1, s-1}=(-1)^{k+1} \operatorname{Li}_{r}(\bar{u}) \operatorname{Li}_{s}(\bar{v})+(-1)^{r-1} \operatorname{Li}_{r}(\bar{u}) \operatorname{Li}_{s}(v)+(-1)^{k} \operatorname{Li}_{k}(\overline{u v}) } \\
& {\left[R_{\bar{u}, u v}(x-y,-y)\right]_{r-1, s-1}=}(-1)^{r} \sum_{m=1}^{k-1}\left(\begin{array}{c}
m-1 \\
r-1
\end{array}\right) \operatorname{Li}_{m}(\bar{u}) \operatorname{Li}_{k-m}(u v) \\
&+(-1)^{s} \sum_{m=1}^{k-1}\left(\begin{array}{c}
m-1 \\
r-1
\end{array}\right)(-1)^{m} \operatorname{Li}_{m}(\bar{u}) \operatorname{Li}_{k-m}(\overline{u v}) \\
&+(-1)^{r-1}\left(\begin{array}{c}
k-1 \\
s-1
\end{array}\right) \operatorname{Li}_{k}(v) \\
& {\left[R_{v, \overline{u v}}(x-y, x)\right]_{r-1, s-1}=}(-1)^{r} \sum_{m=1}^{k-1}\left(\begin{array}{c}
m-1 \\
s-1
\end{array}\right) \operatorname{Li}_{m}(v) \operatorname{Li}_{k-m}(\overline{u v}) \\
&+(-1)^{s} \sum_{m=1}^{k-1}\left(\begin{array}{c}
m-1 \\
s-1
\end{array}\right)(-1)^{m} \operatorname{Li}_{m}(v) \operatorname{Li}_{k-m}(u v) \\
&+(-1)^{r-1}\left(\begin{array}{c}
k-1 \\
r-1
\end{array}\right) \operatorname{Li}_{k}(\bar{u}) .
\end{aligned}
$$

By putting everything together, we recover the statement of Theorem 3

\section{Some Particular CASES}

In this section we consider some particular cases of Theorem 3 that have specific applications beyond the world of multiple zetas and polylogarithms.

Our first result comes from considering $u, v= \pm i$. It is a generalisation of a result in [17] and can be used to simplify some Mahler measure formulas [16, 19]. The specific connection with Mahler measure will be explained in Section 5 
Corollary 5. Let $r, s$ be positive integers such that $k=r+s$ is even. Then

$$
\begin{aligned}
\sum_{0 \leq j<\ell} \frac{(-1)^{j+\ell}}{(2 j+1)^{r} \ell^{s}} & \\
= & -\frac{\left(1+(-1)^{s}\right)}{2}\left(1-2^{1-s}\right) \zeta(s) L\left(\chi_{-4}, r\right)+(-1)^{s} 2^{s-1}\left(\begin{array}{c}
k-1 \\
r-1
\end{array}\right) L\left(\chi_{-4}, k\right) \\
& -(-1)^{s} 2^{s} \sum_{\substack{m=1 \\
m \text { even }}}^{k-1}\left(\begin{array}{c}
m-1 \\
s-1
\end{array}\right)\left(1-2^{-k+m}\right) \zeta(k-m) L\left(\chi_{-4}, m\right) \\
& -(-1)^{s} 2^{s} \sum_{\substack{m=1 \\
m \text { even }}}^{k-1}\left(\begin{array}{c}
m-1 \\
r-1
\end{array}\right) 2^{-k+m} \zeta(k-m) L\left(\chi_{-4}, m\right)
\end{aligned}
$$

Proof. We may write the double series as a sum of imaginary parts of polylogarithms.

$$
\frac{i}{2^{s-2}} \sum_{0 \leq j<\ell} \frac{(-1)^{j+\ell}}{(2 j+1)^{r} \ell^{s}}=2 i \operatorname{Im}\left(\operatorname{Li}_{r, s}(i, i)\right)+2 i \operatorname{Im}\left(\operatorname{Li}_{r, s}(i,-i)\right)
$$

Given the above equation, it suffices to fix $u=i$ and consider $v= \pm i$ in Theorem 3 For $v=i$, Theorem 3 gives

$$
\begin{aligned}
2 \operatorname{Re}_{k}\left(\operatorname{Li}_{r, s}(i, i)\right)= & (-1)^{k} \operatorname{Li}_{k}(-1)+(-1)^{k+1} \operatorname{Li}_{r}(-i) \operatorname{Li}_{s}(-i)+(-1)^{r-1} \operatorname{Li}_{r}(-i) \operatorname{Li}_{s}(i) \\
+ & (-1)^{r-1}\left(\left(\begin{array}{c}
k-1 \\
r-1
\end{array}\right) \operatorname{Li}_{k}(-i)+\left(\begin{array}{c}
k-1 \\
s-1
\end{array}\right) \operatorname{Li}_{k}(i)\right) \\
& +\sum_{m=1}^{k-1}\left(\left(\begin{array}{c}
m-1 \\
r-1
\end{array}\right) \operatorname{Li}_{m}(-i)+\left(\begin{array}{c}
m-1 \\
s-1
\end{array}\right)(-1)^{k+m} \operatorname{Li}_{m}(i)\right) \\
& \times\left((-1)^{r} \operatorname{Li}_{k-m}(-1)+(-1)^{s+m} \operatorname{Li}_{k-m}(-1)\right) .
\end{aligned}
$$

Now consider the case of $v=-i$.

$$
\begin{aligned}
2 \operatorname{Re}_{k}\left(\operatorname{Li}_{r, s}(i,-i)\right)= & (-1)^{k} \operatorname{Li}_{k}(1)+(-1)^{k+1} \operatorname{Li}_{r}(-i) \operatorname{Li}_{s}(i)+(-1)^{r-1} \operatorname{Li}_{r}(-i) \operatorname{Li}_{s}(-i) \\
+ & (-1)^{r-1}\left(\left(\begin{array}{c}
k-1 \\
r-1
\end{array}\right) \operatorname{Li}_{k}(-i)+\left(\begin{array}{c}
k-1 \\
s-1
\end{array}\right) \operatorname{Li}_{k}(-i)\right) \\
+ & \sum_{m=1}^{k-1}\left(\left(\begin{array}{c}
m-1 \\
r-1
\end{array}\right) \operatorname{Li}_{m}(-i)+\left(\begin{array}{c}
m-1 \\
s-1
\end{array}\right)(-1)^{k+m} \operatorname{Li}_{m}(-i)\right) \\
& \times\left((-1)^{r} \operatorname{Li}_{k-m}(1)+(-1)^{s+m} \operatorname{Li}_{k-m}(1)\right) .
\end{aligned}
$$


We will need to restrict to the case of $k$ even so that $\operatorname{Re}_{k}=\operatorname{Im}$. Adding (13) and 14 yields

$$
\begin{aligned}
& \frac{i}{2^{s-2}} \sum_{0 \leq j<\ell} \frac{(-1)^{j+\ell}}{(2 j+1)^{r} \ell^{s}} \\
&=\left(\operatorname{Li}_{k}(1)+\operatorname{Li}_{k}(-1)\right)-\left(1+(-1)^{s}\right) \operatorname{Li}_{r}(-i)\left(\operatorname{Li}_{s}(i)+\operatorname{Li}_{s}(-i)\right) \\
&+(-1)^{s-1} 2\left(\begin{array}{c}
k-1 \\
r-1
\end{array}\right) \operatorname{Li}_{k}(-i)+(-1)^{s-1}\left(\begin{array}{c}
k-1 \\
s-1
\end{array}\right)\left(\operatorname{Li}_{k}(i)+\operatorname{Li}_{k}(-i)\right) \\
&+(-1)^{s} 2 \sum_{\substack{m=1 \\
m \text { even }}}^{k-1}\left(\begin{array}{c}
m-1 \\
s-1
\end{array}\right)\left(\operatorname{Li}_{k-m}(1) \operatorname{Li}_{m}(-i)+\operatorname{Li}_{k-m}(-1) \operatorname{Li}_{m}(i)\right) \\
&+(-1)^{s} 2 \sum_{\substack{m=1 \\
m \text { even }}}^{k-1}\left(\begin{array}{c}
m-1 \\
r-1
\end{array}\right) \operatorname{Li}_{m}(-i)\left(\operatorname{Li}_{k-m}(1)+\operatorname{Li}_{k-m}(-1)\right)
\end{aligned}
$$

The formula above can be greatly simplified. Recall the following formulas that were mentioned earlier in the introduction:

$$
\operatorname{Li}_{n}(1)=\zeta(n), \quad \operatorname{Li}_{n}(-1)=\left(2^{1-n}-1\right) \zeta(n),
$$

and

$$
\operatorname{Li}_{n}(i)=\left(2^{1-2 n}-2^{-n}\right) \zeta(n)+i L\left(\chi_{-4}, n\right) .
$$

We proceed to replace the length-one polylogarithms by the Riemann zeta function and the Dirichlet $L$-function in $\chi_{-4}$. We obtain

$$
\begin{aligned}
& \frac{i}{2^{s-2}} \sum_{0 \leq j<\ell} \frac{(-1)^{j+\ell}}{(2 j+1)^{r} \ell^{s}} \\
& =2^{1-k} \zeta(k)-\left(1+(-1)^{s}\right)\left(\left(2^{1-2 r}-2^{-r}\right) \zeta(r)-i L\left(\chi_{-4}, r\right)\right) 2\left(2^{1-2 s}-2^{-s}\right) \zeta(s) \\
& +(-1)^{s-1} 2\left(\begin{array}{c}
k-1 \\
r-1
\end{array}\right)\left(\left(2^{1-2 k}-2^{-k}\right) \zeta(k)-i L\left(\chi_{-4}, k\right)\right) \\
& +(-1)^{s-1}\left(\begin{array}{c}
k-1 \\
s-1
\end{array}\right) 2\left(2^{1-2 k}-2^{-k}\right) \zeta(k) \\
& +(-1)^{s} 2 \sum_{\substack{m=1 \\
m \text { even }}}^{k-1}\left(\begin{array}{c}
m-1 \\
s-1
\end{array}\right) \zeta(k-m)\left(\left(2^{1-2 m}-2^{-m}\right) \zeta(m)-i L\left(\chi_{-4}, m\right)\right) \\
& +(-1)^{s} 2 \sum_{\substack{m=1 \\
m \text { even }}}^{k-1}\left(\begin{array}{c}
m-1 \\
s-1
\end{array}\right)\left(2^{1-k+m}-1\right) \zeta(k-m)\left(\left(2^{1-2 m}-2^{-m}\right) \zeta(m)+i L\left(\chi_{-4}, m\right)\right) \\
& +(-1)^{s} 2 \sum_{\substack{m=1 \\
m \text { even }}}^{k-1}\left(\begin{array}{c}
m-1 \\
r-1
\end{array}\right)\left(\left(2^{1-2 m}-2^{-m}\right) \zeta(m)-i L\left(\chi_{-4}, m\right)\right) 2^{1-k+m} \zeta(k-m) .
\end{aligned}
$$


Since the left-hand side is purely imaginary, the same must be true for the right-hand side. Thus, we ignore the real terms in the equation and we get

$$
\begin{aligned}
\frac{i}{2^{s-2}} \sum_{0 \leq j<\ell} \frac{(-1)^{j+\ell}}{(2 j+1)^{r} \ell^{s}} \\
=\left(1+(-1)^{s}\right) 2\left(2^{1-2 s}-2^{-s}\right) \zeta(s) i L\left(\chi_{-4}, r\right)+(-1)^{s} 2\left(\begin{array}{c}
k-1 \\
r-1
\end{array}\right) i L\left(\chi_{-4}, k\right) \\
\quad+(-1)^{s} 2^{2} \sum_{\substack{m=1 \\
m \text { even }}}^{k-1}\left(\begin{array}{c}
m-1 \\
s-1
\end{array}\right)\left(2^{-k+m}-1\right) \zeta(k-m) i L\left(\chi_{-4}, m\right) \\
\quad-(-1)^{s} 2^{2} \sum_{\substack{m=1 \\
m \text { even }}}^{k-1}\left(\begin{array}{c}
m-1 \\
r-1
\end{array}\right) 2^{-k+m} \zeta(k-m) i L\left(\chi_{-4}, m\right)
\end{aligned}
$$

Dividing in both sides by $\frac{i}{2^{s-2}}$ we recover the desired result.

We remark that since the right-hand side of 17 must be purely imaginary, we must have that

$$
\begin{aligned}
0= & 2^{1-k} \zeta(k)+(-1)^{s-1} 2\left(\begin{array}{c}
k \\
s
\end{array}\right)\left(2^{1-2 k}-2^{-k}\right) \zeta(k) \\
& -\left(1+(-1)^{s}\right) 2\left(2^{1-2 r}-2^{-r}\right)\left(2^{1-2 s}-2^{-s}\right) \zeta(r) \zeta(s) \\
& +(-1)^{s} 2^{2-k} \sum_{\substack{m=1 \\
m \text { even }}}^{k-1}\left(\left(\begin{array}{c}
m-1 \\
r-1
\end{array}\right)+\left(\begin{array}{c}
m-1 \\
s-1
\end{array}\right)\right)\left(2^{1-m}-1\right) \zeta(m) \zeta(k-m) .
\end{aligned}
$$

By using Euler's identity

$$
\zeta(2 j)=\frac{(-1)^{j+1}(2 \pi)^{2 j}}{2(2 j) !} B_{2 j},
$$

we conclude that for $r, s$ positive integers and $k=r+s$ even,

$$
\begin{aligned}
0= & B_{k}+(-1)^{s-1}\left(\begin{array}{c}
k \\
s
\end{array}\right)\left(2^{1-k}-1\right) B_{k} \\
& +\frac{\left(1+(-1)^{s}\right)}{2}\left(\begin{array}{c}
k \\
s
\end{array}\right)\left(2^{1-r}-1\right)\left(2^{1-s}-1\right) B_{r} B_{s} \\
& +(-1)^{s-1} \sum_{\substack{m=1 \\
m \text { even }}}^{k-1}\left(\left(\begin{array}{c}
m-1 \\
r-1
\end{array}\right)+\left(\begin{array}{c}
m-1 \\
s-1
\end{array}\right)\right)\left(\begin{array}{c}
k \\
m
\end{array}\right)\left(2^{1-m}-1\right) B_{m} B_{k-m} .
\end{aligned}
$$

In the formula above, if $r$ and $s$ are even, it is correct to replace Euler's identity (18) for $\zeta(r)$ and for $\zeta(s)$. If they are both odd, the corresponding term in the second line vanishes since $1+(-1)^{s}=0$. Thus, the equality remains valid. Equation $(19)$ can be independently proved by setting $x=1 / 2$ in [23, eq. (8), p. 75] (see also [1, eq. (19b), p. 276]).

A similar sum can also be computed by means of Theorem 3 
Corollary 6. Let $r, s$ be positive integers such that $k=r+s$ is odd. Then

$$
\begin{aligned}
& \sum_{0 \leq j<\ell} \frac{(-1)^{j+\ell}}{(2 j+1)^{r}(2 \ell+1)^{s}} \\
& =\frac{1}{2}\left(2^{-k}-1\right) \zeta(k)+\frac{1+(-1)^{r}}{2} L\left(\chi_{-4}, r\right) L\left(\chi_{-4}, s\right) \\
& \quad+(-1)^{r} 2^{-k} \sum_{\substack{m=1 \\
m \text { odd }}}^{k-1}\left(\left(\begin{array}{c}
m-1 \\
r-1
\end{array}\right)+\left(\begin{array}{c}
m-1 \\
s-1
\end{array}\right)\right)\left(2^{1-m}-1\right)\left(2^{k-m}-1\right) \zeta(m) \zeta(k-m) .
\end{aligned}
$$

Proof. This double alternating series may also be written in terms of polylogarithms:

$$
\sum_{0 \leq j<\ell} \frac{(-1)^{j+\ell}}{(2 j+1)^{r}(2 \ell+1)^{s}}=\frac{1}{2^{k}} \operatorname{Li}_{r, s}(-1,-1)-\operatorname{Re}\left(\operatorname{Li}_{r, s}(i, i)\right) .
$$

We return to equation (13), this time for $k$ odd since we need the real part $\operatorname{Re}\left(\operatorname{Li}_{r, s}(i, i)\right)$, and we combine this with Theorem 2 using $\rho=\sigma=-1$. We obtain

$$
\begin{aligned}
& \sum_{0 \leq j<\ell} \frac{(-1)^{j+\ell}}{(2 j+1)^{r}(2 \ell+1)^{s}} \\
&=-2^{-k-1} \operatorname{Li}_{k}(1)+\left(1+(-1)^{s}\right) 2^{-k-1} \operatorname{Li}_{r}(-1) \operatorname{Li}_{s}(-1) \\
&+(-1)^{s} 2^{-k-1}\left(\begin{array}{c}
k \\
s
\end{array}\right) \operatorname{Li}_{k}(-1) \\
&-(-1)^{s} 2^{-k} \sum_{m=1}^{k-1}\left(\left(\begin{array}{c}
m-1 \\
r-1
\end{array}\right)+\left(\begin{array}{c}
m-1 \\
s-1
\end{array}\right)\right) \operatorname{Li}_{m}(-1) \operatorname{Li}_{k-m}(1) \\
&+\frac{1}{2} \operatorname{Li}_{k}(-1)-\frac{1}{2} \operatorname{Li}_{r}(-i) \operatorname{Li}_{s}(-i)+\frac{(-1)^{r}}{2} \operatorname{Li}_{r}(-i) \operatorname{Li}_{s}(i) \\
&+\frac{(-1)^{r}}{2}\left(\left(\begin{array}{c}
k-1 \\
r-1
\end{array}\right) \operatorname{Li}_{k}(-i)+\left(\begin{array}{c}
k-1 \\
s-1
\end{array}\right) \operatorname{Li}_{k}(i)\right) \\
&-(-1)^{r} \sum_{\substack{m=1 \\
m \text { odd }}}^{k-1}\left(\left(\begin{array}{c}
m-1 \\
r-1
\end{array}\right) \operatorname{Li}_{m}(-i)+\left(\begin{array}{c}
m-1 \\
s-1
\end{array}\right) \operatorname{Li}_{m}(i)\right) \operatorname{Li}_{k-m}(-1)
\end{aligned}
$$

With the aid of formulas $(15)$ and $(16)$, we replace the length-one polylogarithms in the above equation by the Riemann zeta function and the Dirichlet $L$-function in $\chi_{-4}$. 


$$
\begin{aligned}
& \sum_{0 \leq j<\ell} \frac{(-1)^{j+\ell}}{(2 j+1)^{r}(2 \ell+1)^{s}} \\
& =-2^{-k-1} \zeta(k)+\left(1+(-1)^{s}\right) 2^{-k-1}\left(2^{1-r}-1\right) \zeta(r)\left(2^{1-s}-1\right) \zeta(s) \\
& +(-1)^{s} 2^{-k-1}\left(\begin{array}{l}
k \\
s
\end{array}\right)\left(2^{1-k}-1\right) \zeta(k) \\
& -(-1)^{s} 2^{-k} \sum_{\substack{m=1 \\
m \text { odd }}}^{k-1}\left(\left(\begin{array}{c}
m-1 \\
r-1
\end{array}\right)+\left(\begin{array}{c}
m-1 \\
s-1
\end{array}\right)\right)\left(2^{1-m}-1\right) \zeta(m) \zeta(k-m) \\
& +\frac{1}{2}\left(2^{1-k}-1\right) \zeta(k) \\
& -\frac{1}{2}\left(\left(2^{1-2 r}-2^{-r}\right) \zeta(r)-i L\left(\chi_{-4}, r\right)\right)\left(\left(2^{1-2 s}-2^{-s}\right) \zeta(s)-i L\left(\chi_{-4}, s\right)\right) \\
& +\frac{(-1)^{r}}{2}\left(\left(2^{1-2 r}-2^{-r}\right) \zeta(r)-i L\left(\chi_{-4}, r\right)\right)\left(\left(2^{1-2 s}-2^{-s}\right) \zeta(s)+i L\left(\chi_{-4}, s\right)\right) \\
& +\frac{(-1)^{r}}{2}\left(\left(\begin{array}{c}
k-1 \\
r-1
\end{array}\right)\left(\left(2^{1-2 k}-2^{-k}\right) \zeta(k)-i L\left(\chi_{-4}, k\right)\right)\right. \\
& \left.+\left(\begin{array}{c}
k-1 \\
s-1
\end{array}\right)\left(\left(2^{1-2 k}-2^{-k}\right) \zeta(k)+i L\left(\chi_{-4}, k\right)\right)\right) \\
& -(-1)^{r} \sum_{\substack{m=1 \\
m \text { odd }}}^{k-1}\left(\begin{array}{c}
m-1 \\
r-1
\end{array}\right)\left(\left(2^{1-2 m}-2^{-m}\right) \zeta(m)-i L\left(\chi_{-4}, m\right)\right)\left(2^{1-k+m}-1\right) \zeta(k-m) \\
& -(-1)^{r} \sum_{\substack{m=1 \\
m \text { odd }}}^{k-1}\left(\begin{array}{c}
m-1 \\
s-1
\end{array}\right)\left(\left(2^{1-2 m}-2^{-m}\right) \zeta(m)+i L\left(\chi_{-4}, m\right)\right)\left(2^{1-k+m}-1\right) \zeta(k-m) \text {. }
\end{aligned}
$$

Since the left-hand side is real, the same must be true for the right-hand side. Therefore we take the real terms and simplify as much as possible:

$$
\begin{aligned}
& \sum_{0 \leq j<\ell} \frac{(-1)^{j+\ell}}{(2 j+1)^{r}(2 \ell+1)^{s}} \\
& =\frac{1}{2}\left(2^{-k}-1\right) \zeta(k)+\frac{1+(-1)^{r}}{2} L\left(\chi_{-4}, r\right) L\left(\chi_{-4}, s\right) \\
& \quad+(-1)^{r} 2^{-k} \sum_{\substack{m=1 \\
m \text { odd }}}^{k-1}\left(\left(\begin{array}{c}
m-1 \\
r-1
\end{array}\right)+\left(\begin{array}{c}
m-1 \\
s-1
\end{array}\right)\right)\left(2^{1-m}-1\right)\left(2^{k-m}-1\right) \zeta(m) \zeta(k-m)
\end{aligned}
$$

This recovers the result. 
Going back to 20 , we have that the imaginary part of the right-hand side must be zero. This yields

$$
\begin{aligned}
0= & \frac{1+(-1)^{r}}{2} L\left(\chi_{-4}, s\right)\left(2^{1-2 r}-2^{-r}\right) \zeta(r)+\frac{1-(-1)^{r}}{2} L\left(\chi_{-4}, r\right)\left(2^{1-2 s}-2^{-s}\right) \zeta(s) \\
& -\frac{(-1)^{r}}{2}\left(\left(\begin{array}{c}
k-1 \\
r-1
\end{array}\right)-\left(\begin{array}{c}
k-1 \\
s-1
\end{array}\right)\right) L\left(\chi_{-4}, k\right) \\
& +(-1)^{r} \sum_{\substack{m=1 \\
m \text { odd }}}^{k-1}\left(\left(\begin{array}{c}
m-1 \\
r-1
\end{array}\right)-\left(\begin{array}{c}
m-1 \\
s-1
\end{array}\right)\right) L\left(\chi_{-4}, m\right)\left(2^{1-k+m}-1\right) \zeta(k-m) .
\end{aligned}
$$

Odd values of the Dirichlet $L$ function in $\chi_{-4}$ are given by Euler numbers:

$$
L\left(\chi_{-4}, 2 j+1\right)=(-1)^{j} \frac{E_{2 j}}{2(2 j) !}\left(\frac{\pi}{2}\right)^{2 j+1} .
$$

By replacing (21) as well as Euler's identity 18 for $\zeta(2 j)$ in the previous equation we obtain

$$
\begin{aligned}
0= & \frac{1+(-1)^{r}}{2}\left(\begin{array}{c}
k-1 \\
s-1
\end{array}\right)\left(2-2^{r}\right) E_{s-1} B_{r}+\frac{1-(-1)^{r}}{2}\left(\begin{array}{l}
k-1 \\
r-1
\end{array}\right)\left(2-2^{s}\right) E_{r-1} B_{s} \\
& +(-1)^{r}\left(\left(\begin{array}{c}
k-1 \\
r-1
\end{array}\right)-\left(\begin{array}{c}
k-1 \\
s-1
\end{array}\right)\right) E_{k-1} \\
& +(-1)^{r} \sum_{\substack{m=1 \\
m \text { odd }}}^{k-1}\left(\left(\begin{array}{c}
m-1 \\
r-1
\end{array}\right)-\left(\begin{array}{c}
m-1 \\
s-1
\end{array}\right)\right)\left(\begin{array}{c}
k-1 \\
m-1
\end{array}\right) 2^{2 k-2 m}\left(2^{1-k+m}-1\right) E_{m-1} B_{k-m} .
\end{aligned}
$$

In the computation above, since $k$ is odd, exactly one between $r$ and $s$ is even and the other is odd. It is correct to replace identities (18) and (21) for $\zeta(r), \zeta(s)$, $L\left(\chi_{-4}, r\right)$ and $L\left(\chi_{-4}, s\right)$ in the first line. Indeed the term with the wrong parity vanishes according to the parity of $r$ due to the factors $1+(-1)^{r}$ and $1-(-1)^{r}$. Thus, the equality remains valid. We are not aware of a reference for this formula, but we expect that it can be proved independently by considering relationships between Euler polynomials and Bernoulli numbers, in a similar fashion as equation (19).

The following result will be useful in Section 5 
Corollary 7. Let $r, s$ be positive integers such that $k=r+s$ is even. Then

$$
\begin{aligned}
\sum_{0<j \leq \ell} \frac{(-1)^{j+\ell}}{j^{r}(2 \ell+1)^{s}} & \\
= & -\frac{\left(1-(-1)^{r}\right)}{2}\left(1-2^{1-r}\right) \zeta(r) L\left(\chi_{-4}, s\right)-(-1)^{r} 2^{r-1}\left(\begin{array}{c}
k-1 \\
s-1
\end{array}\right) L\left(\chi_{-4}, k\right) \\
& +(-1)^{r} 2^{r} \sum_{\substack{m=1 \\
m \text { even }}}^{k-1}\left(\begin{array}{c}
m-1 \\
r-1
\end{array}\right)\left(1-2^{-k+m}\right) \zeta(k-m) L\left(\chi_{-4}, m\right) \\
& +(-1)^{r} 2^{r} \sum_{\substack{m=1 \\
m \text { even }}}^{k-1}\left(\begin{array}{c}
m-1 \\
s-1
\end{array}\right) 2^{-k+m} \zeta(k-m) L\left(\chi_{-4}, m\right)
\end{aligned}
$$

Proof. Notice that

$$
\begin{aligned}
\left(2^{1-s}-1\right) \zeta(s) L\left(\chi_{-4}, r\right) & =\left(\sum_{\ell=1}^{\infty} \frac{(-1)^{\ell}}{\ell^{s}}\right)\left(\sum_{j=0}^{\infty} \frac{(-1)^{j}}{(2 j+1)^{r}}\right) \\
& =\sum_{0 \leq j<\ell} \frac{(-1)^{j+\ell}}{(2 j+1)^{r} \ell^{s}}+\sum_{0<\ell \leq j} \frac{(-1)^{j+\ell}}{(2 j+1)^{r} \ell^{s}}
\end{aligned}
$$

Switching $j$ and $\ell$ in the last sum, we get

$$
\sum_{0<j \leq \ell} \frac{(-1)^{j+\ell}}{j^{s}(2 \ell+1)^{r}}=\left(2^{1-s}-1\right) \zeta(s) L\left(\chi_{-4}, r\right)-\sum_{0 \leq j<\ell} \frac{(-1)^{j+\ell}}{(2 j+1)^{r} \ell^{s}}
$$

and the result follows from Corollary 5 and switching $r$ with $s$.

For the next application we are going to look at some polylogarithms arising from evaluations of Feynman diagrams (see [7]). Before proceeding any further, we recall the definition of the Dirichlet $L$-function in the character of conductor 3 , given by

$$
L\left(\chi_{-3}, s\right):=\sum_{j=1}^{\infty} \frac{\chi_{-3}(j)}{j^{s}}, \quad \chi_{-3}(j)=\left\{\begin{array}{cl}
1 & j \equiv 1 \bmod 3 \\
-1 & j \equiv-1 \bmod 3 \\
0 & 3 \mid j .
\end{array}\right.
$$

We can prove the following result.

Corollary 8. Let

$$
V_{s, r}=\sum_{0<j<\ell} \frac{(-1)^{\ell} \cos (2 \pi j / 3)}{j^{r} \ell^{s}}
$$

Then, we have

$$
V_{2,1}=-\frac{41}{36} \zeta(3)+\frac{\pi^{2}}{18} \log 3+\frac{\pi}{2 \sqrt{3}} L\left(\chi_{-3}, 2\right)
$$


Let $r$ be even, $s$ odd, and $s>1$. Then,

$$
\begin{aligned}
V_{s, r}= & -\frac{\left(2^{1-k}-1\right)\left(3^{1-k}-1\right)}{4} \zeta(k) \\
& -\left(\left(\begin{array}{c}
k-1 \\
r-1
\end{array}\right) \frac{3^{1-k}-1}{4} \zeta(k)+\left(\begin{array}{c}
k-1 \\
s-1
\end{array}\right) \frac{2^{1-k}-1}{2} \zeta(k)\right) \\
& -\frac{3}{4} \sum_{\substack{m=1 \\
m \text { even }}}^{k-1}\left(\begin{array}{c}
m-1 \\
r-1
\end{array}\right)\left(2^{1-k+m}+1\right) L\left(\chi_{-3}, m\right) L\left(\chi_{-3}, k-m\right) \\
& +\sum_{\substack{m=1 \\
m-1}}\left(\left(\begin{array}{c}
m-1 \\
r-1
\end{array}\right) \frac{3^{1-m}-1}{4} \zeta(m)+\left(\begin{array}{c}
m-1 \\
s-1
\end{array}\right) \frac{2^{1-m}-1}{2} \zeta(m)\right) \\
& \quad \times\left(2^{1-k+m}-1\right)\left(3^{1-k+m}-1\right) \zeta(k-m) .
\end{aligned}
$$

We remark that the case of $V_{2,1}$ is formula (163) in [7]. We will see that Theorem 3 applies in this case as long as $k=r+s$ is odd. The additional conditions on $r$ and $s$ are only there to simplify the final formula.

Proof. Let $\omega_{3}=\frac{-1+\sqrt{3} i}{2}$ be the third root of unity. Then we can write

$$
\sum_{0<j<\ell} \frac{(-1)^{\ell} \cos (2 \pi j / 3)}{j^{r} \ell^{s}}=\operatorname{Re}\left(\operatorname{Li}_{r, s}\left(\omega_{3},-1\right)\right) .
$$

We set $u=\omega_{3}$ and $v=-1$. Assume also that $k$ is odd so that $\operatorname{Re}_{k}$ corresponds to Re. By Theorem 3 ,

$$
\begin{aligned}
2 \operatorname{Re}\left(\operatorname{Li}_{r, s}\left(\omega_{3},-1\right)\right)= & -\operatorname{Li}_{k}\left(-\overline{\omega_{3}}\right)+\operatorname{Li}_{r}\left(\overline{\omega_{3}}\right) \operatorname{Li}_{s}(-1)+(-1)^{r-1} \operatorname{Li}_{r}\left(\overline{\omega_{3}}\right) \operatorname{Li}_{s}(-1) \\
+ & (-1)^{r-1}\left(\left(\begin{array}{c}
k-1 \\
r-1
\end{array}\right) \operatorname{Li}_{k}\left(\overline{\omega_{3}}\right)+\left(\begin{array}{c}
k-1 \\
s-1
\end{array}\right) \operatorname{Li}_{k}(-1)\right) \\
+ & \sum_{m=1}^{k-1}\left(\left(\begin{array}{c}
m-1 \\
r-1
\end{array}\right) \operatorname{Li}_{m}\left(\overline{\omega_{3}}\right)+\left(\begin{array}{c}
m-1 \\
s-1
\end{array}\right)(-1)^{1+m} \operatorname{Li}_{m}(-1)\right) \\
& \times\left((-1)^{r} \operatorname{Li}_{k-m}\left(-\omega_{3}\right)+(-1)^{r+1+m} \operatorname{Li}_{k-m}\left(-\overline{\omega_{3}}\right)\right) .
\end{aligned}
$$

In order to simplify the above equality, we need to express polylogarithms of length one in third and sixth roots of unity in terms of $\zeta(n), L\left(\chi_{-3}, n\right)$ and elementary functions. For $n \geq 2$ we have

$$
\begin{aligned}
\operatorname{Li}_{n}\left(\omega_{3}\right) & =\frac{3^{1-n}-1}{2} \zeta(n)+i \frac{\sqrt{3}}{2} L\left(\chi_{-3}, n\right), \\
\operatorname{Li}_{n}\left(-\omega_{3}\right) & =\frac{\left(2^{1-n}-1\right)\left(3^{1-n}-1\right)}{2} \zeta(n)-i \frac{\sqrt{3}\left(2^{1-n}+1\right)}{2} L\left(\chi_{-3}, n\right) .
\end{aligned}
$$


For $n=1$, we have

$$
\operatorname{Li}_{1}\left(\omega_{3}\right)=-\frac{\log 3}{2}+i \frac{\pi}{6}, \quad \operatorname{Li}_{1}\left(-\omega_{3}\right)=-i \frac{\pi}{3} .
$$

We now replace the above equalities in 22 . First assume that $r=1, s=2$. Then,

$$
\begin{aligned}
2 \operatorname{Re}\left(\operatorname{Li}_{1,2}\left(\omega_{3},-1\right)\right)= & -\operatorname{Li}_{3}\left(-\overline{\omega_{3}}\right)+2 \operatorname{Li}_{1}\left(\overline{\omega_{3}}\right) \operatorname{Li}_{2}(-1)+\operatorname{Li}_{3}\left(\overline{\omega_{3}}\right)+2 \operatorname{Li}_{3}(-1) \\
& -\operatorname{Li}_{1}\left(\overline{\omega_{3}}\right)\left(\operatorname{Li}_{2}\left(-\omega_{3}\right)+\operatorname{Li}_{2}\left(-\overline{\omega_{3}}\right)\right) \\
& +\left(\operatorname{Li}_{2}\left(\overline{\omega_{3}}\right)-\operatorname{Li}_{2}(-1)\right)\left(-\operatorname{Li}_{1}\left(-\omega_{3}\right)+\operatorname{Li}_{1}\left(-\overline{\omega_{3}}\right)\right) \\
= & -\frac{41}{18} \zeta(3)+\frac{\pi^{2}}{9} \log 3+\frac{\pi}{\sqrt{3}} L\left(\chi_{-3}, 2\right)
\end{aligned}
$$

and we recover the formula for $V_{2,1}$.

Now assume that $r$ is even, $s$ odd, and $s>1$. In particular, we have that $k$ is odd. We obtain

$$
\begin{aligned}
2 \operatorname{Re}\left(\operatorname{Li}_{r, s}\left(\omega_{3},-1\right)\right)= & -\frac{\left(2^{1-k}-1\right)\left(3^{1-k}-1\right)}{2} \zeta(k) \\
& -\left(\left(\begin{array}{c}
k-1 \\
r-1
\end{array}\right) \frac{3^{1-k}-1}{2} \zeta(k)+\left(\begin{array}{c}
k-1 \\
s-1
\end{array}\right)\left(2^{1-k}-1\right) \zeta(k)\right) \\
& -\frac{3}{2} \sum_{\substack{m=1 \\
m \text { even }}}^{k-1}\left(\begin{array}{c}
m-1 \\
r-1
\end{array}\right)\left(2^{1-k+m}+1\right) L\left(\chi_{-3}, m\right) L\left(\chi_{-3}, k-m\right) \\
& +\sum_{\substack{m=1 \\
m \text { odd }}}^{k-1}\left(\left(\begin{array}{c}
m-1 \\
r-1
\end{array}\right) \frac{3^{1-m}-1}{2} \zeta(m)+\left(\begin{array}{c}
m-1 \\
s-1
\end{array}\right)\left(2^{1-m}-1\right) \zeta(m)\right) \\
& \times\left(2^{1-k+m}-1\right)\left(3^{1-k+m}-1\right) \zeta(k-m) .
\end{aligned}
$$

This concludes the proof of the formula for $V_{s, r}$.

\section{Applications to Mahler measure}

We present in this section two applications of Corollary 5 and Corollary 7 to Mahler measure. These applications were the main motivation for [17] and this paper.

5.1. A Mahler measure example. Our most important application comes from formula (4) in Theorem 1 in [16] which claims

$$
\begin{aligned}
& \mathrm{m}(1+x+\left.\left(\frac{1-x_{1}}{1+x_{1}}\right) \cdots\left(\frac{1-x_{2 n+1}}{1+x_{2 n+1}}\right)(1+y) z\right) \\
&=\sum_{h=0}^{n} \frac{s_{n-h}\left(1^{2}, 3^{2}, \ldots,(2 n-1)^{2}\right)}{(2 n) !}\left(\frac{2}{\pi}\right)^{2 h+3} \\
& \quad \times \frac{1}{4}\left(i(2 h) ! \mathcal{L}_{3,2 h+1}(i, i)+(2 h+1) ! \pi^{2} L\left(\chi_{-4}, 2 h+2\right)\right) .
\end{aligned}
$$


Here the $s_{l}\left(a_{1}, \ldots, a_{k}\right)$ coefficients are given by

$$
s_{l}\left(a_{1}, \ldots, a_{k}\right)= \begin{cases}1 & \text { if } l=0 \\ \sum_{i_{1}<\cdots<i_{l}} a_{i_{1}} \cdots a_{i_{l}} & \text { if } 0<l \leq k \\ 0 & \text { if } k<l\end{cases}
$$

and $\mathcal{L}_{3,2 h+1}(i, i)$ is a combination of multiple polylogarithms that arises when computing a certain type of iterated integral. It is given as follows:

$$
\begin{aligned}
\mathcal{L}_{r, s}(i, i) & :=2\left(\operatorname{Li}_{r, s}(i, i)-\operatorname{Li}_{r, s}(-i, i)+\operatorname{Li}_{r, s}(i,-i)-\operatorname{Li}_{r, s}(-i,-i)\right) \\
& =2 \sum_{0<j<\ell} \frac{i^{j} i^{\ell}-(-i)^{j} i^{\ell}+i^{j}(-i)^{\ell}-(-i)^{j}(-i)^{\ell}}{j^{r} \ell^{s}} \\
& =2 \sum_{0<j<\ell} \frac{i^{j+\ell}\left(1+(-1)^{j+1}+(-1)^{\ell}+(-1)^{\ell+j+1}\right)}{j^{r} \ell^{s}} \\
& =2 \sum_{\substack{0<j<\ell \\
\ell \text { odd }}} \frac{4 i^{j+\ell}}{j^{r} \ell^{s}} \\
& =2 \sum_{0<2 j+1<2 \ell} \frac{4 i^{2 j+2 \ell+1}}{(2 j+1)^{r}(2 \ell)^{s}} \\
& =\frac{i}{2^{s-3}} \sum_{0 \leq j<\ell} \frac{(-1)^{j+\ell}}{(2 j+1)^{r} \ell^{s}} .
\end{aligned}
$$

Therefore, we have

$$
\mathcal{L}_{3,2 h+1}(i, i)=\frac{i}{2^{2 h-2}} \sum_{0 \leq j<\ell} \frac{(-1)^{j+\ell}}{(2 j+1)^{3} \ell^{2 h+1}}
$$

The main result of [17] allowed the first named author to compute this sum for $h=0$ and simplify the first case of formula (1), which first appeared in [15]. In this way, a closed formula in terms of the Riemann zeta function and the Dirichlet $L$-function in the character of conductor 4 was obtained for $n=0$.

Since $3+2 h+1$ is even, we can now use Corollary 5 to compute $\mathcal{L}_{3,2 h+1}(i, i)$ for any $h$. We obtain

$$
\begin{aligned}
-i \mathcal{L}_{3,2 h+1}(i, i)= & -2(2 h+3)(2 h+2) L\left(\chi_{-4}, 2 h+4\right) \\
& +(2 h+1) \pi^{2} L\left(\chi_{-4}, 2 h+2\right) \\
& +8 \sum_{n=1}^{h}\left(\begin{array}{c}
2 h+3-2 n \\
2
\end{array}\right) \frac{\zeta(2 n)}{2^{2 n}} L\left(\chi_{-4}, 2 h+4-2 n\right) .
\end{aligned}
$$


Combining the result above with (23) yields the following formula as presented without proof in [19]:

$$
\begin{aligned}
\mathrm{m}(1+ & \left.x+\left(\frac{1-x_{1}}{1+x_{1}}\right) \cdots\left(\frac{1-x_{2 n+1}}{1+x_{2 n+1}}\right)(1+y) z\right) \\
= & \sum_{h=0}^{n} \frac{s_{n-h}\left(1^{2}, 3^{2}, \ldots,(2 n-1)^{2}\right)}{(2 n) !}\left(\frac{2}{\pi}\right)^{2 h+3} \\
& \left.\times \sum_{\ell=0}^{h}\left(\begin{array}{c}
2 h+1 \\
2 \ell
\end{array}\right) \frac{(2 h+3-2 \ell) !}{2(2 h+1)}(-1)^{\ell} B_{2 \ell} \pi^{2 \ell} L\left(\chi_{-4}, 2 h+4-2 \ell\right)\right) .
\end{aligned}
$$

5.2. A higher Mahler measure example. For $k$ a positive integer, the $k$-higher Mahler measure is a generalisation consisting of taking the $k$-th power of the logarithm inside the integral:

$$
\mathrm{m}_{k}(P):=\frac{1}{(2 \pi i)^{n}} \int_{\mathbb{T}^{n}} \log ^{k}\left|P\left(x_{1}, \ldots, x_{n}\right)\right| \frac{\mathrm{d} x_{1}}{x_{1}} \cdots \frac{\mathrm{d} x_{n}}{x_{n}} .
$$

This construction also yields special values of $L$-functions, although the connection with Beilinson's conjectures is much less clear and therefore, there is a particular interest in generating examples that may eventually lead to a general theory involving not just the Mahler measure, but its higher counterpart as well.

The authors of this paper showed the following result in [19]:

$$
\begin{aligned}
\mathrm{m}_{3}(z & \left.+\left(\frac{1-x_{1}}{1+x_{1}}\right) \cdots\left(\frac{1-x_{2 n+1}}{1+x_{2 n+1}}\right)\right) \\
= & \sum_{h=0}^{n} \frac{s_{n-h}\left(1^{2}, 3^{2}, \ldots,(2 n-1)^{2}\right)}{(2 n) !}\left(\frac{2}{\pi}\right)^{2 h+1} \\
& \quad \times\left(3 i(2 h) ! \mathcal{L}_{1,2,2 h+1}^{-}(1, i, i)-\frac{3}{2} i(2 h+1) ! \mathcal{L}_{2,2 h+2}^{-}(i, i)+(2 h+3) ! L\left(\chi_{-4}, 2 h+4\right)\right) .
\end{aligned}
$$

On the right hand side of (24) we find, once again, combinations of multiple polylogarithms:

$\mathcal{L}_{n_{1}, \ldots, n_{m}}^{-}\left(z_{1}, \ldots, z_{m}\right)=\sum_{\left(\sigma_{1}, \ldots, \sigma_{m}\right) \in\{0,1\}^{m}}(-1)^{\sigma_{m}} \operatorname{Li}_{n_{1}, \ldots, n_{m}}\left((-1)^{\sigma_{1}} z_{1}, \ldots,(-1)^{\sigma_{m}} z_{m}\right)$.

In particular, we have

$$
\begin{aligned}
\mathcal{L}_{2,2 h+2}^{-}(i, i) & =\operatorname{Li}_{2,2 h+2}(i, i)-\operatorname{Li}_{2,2 h+2}(i,-i)+\operatorname{Li}_{2,2 h+2}(-i, i)-\mathrm{Li}_{2,2 h+2}(-i,-i) \\
& =i \sum_{0<j \leq \ell} \frac{(-1)^{j+\ell}}{j^{2}(2 \ell+1)^{2 h+2}} .
\end{aligned}
$$

Since $2+2 h+2$ is even, we can use Corollary 7 to get

$$
\begin{aligned}
-i \mathcal{L}_{2,2 h+2}^{-}(i, i)= & -(2 h+3)(2 h+2) L\left(\chi_{-4}, 2 h+4\right) \\
& +\zeta(2) L\left(\chi_{-4}, 2 h+2\right) \\
& +4 \sum_{n=1}^{h+1}\left(2^{2 n}-1\right)(2 h+3-2 n) \frac{\zeta(2 n)}{2^{2 n}} L\left(\chi_{-4}, 2 h+4-2 n\right) .
\end{aligned}
$$


This allows us to simplify the formula in the following way:

$$
\begin{aligned}
\mathrm{m}_{3}(z & \left.+\left(\frac{1-x_{1}}{1+x_{1}}\right) \cdots\left(\frac{1-x_{2 n+1}}{1+x_{2 n+1}}\right)\right) \\
=\sum_{h=0}^{n} & \frac{s_{n-h}\left(1^{2}, 3^{2}, \ldots,(2 n-1)^{2}\right)}{(2 n) !}\left(\frac{2}{\pi}\right)^{2 h+1} \\
\quad & \quad\left(3 i(2 h) ! \mathcal{L}_{1,2,2 h+1}^{-}(1, i, i)-\frac{1}{2}(2 h+3) ! L\left(\chi_{-4}, 2 h+4\right)\right. \\
& +\frac{1}{4}(2 h+1) ! \pi^{2} L\left(\chi_{-4}, 2 h+2\right) \\
& \left.\quad+3 \sum_{\ell=1}^{h+1}\left(\begin{array}{c}
2 h+2 \\
2 \ell
\end{array}\right) \frac{(2 h+3-2 \ell) !}{2 h+2}\left(2^{2 \ell}-1\right)(-1)^{\ell-1} B_{2 \ell} \pi^{2 \ell} L\left(\chi_{-4}, 2 h+4-2 \ell\right)\right) .
\end{aligned}
$$

Unfortunately, we do not have a method for further simplification of the term $\mathcal{L}_{1,2,2 h+1}^{-}(1, i, i)$ of length 3 .

\section{FinAL REMARKS}

There are several directions where this work could be continued. The most obvious one is the generalisation of Theorem 3 to the reduction of multiple polylogarithms of higher length. This general aspect seems to be quite involved from the combinatorial point of view. A derived question in this direction is the extension of Proposition 4 to higher length and arbitrary parameters of absolute value one.

Another possible question is the reduction of the opposite parity in Theorem 3 . namely, the real part for weight even and the imaginary part for weight odd. There are several results for specific lower weight where Euler sums have been reduced to combinations of length-one polylogarithms, such as the following formula from [4, where there is a reduction of the real part for even weight (equal to 4):

$$
\operatorname{Li}_{1,3}(1,-1)=2 \mathrm{Li}_{4}\left(\frac{1}{2}\right)+\frac{7 \log 2}{4} \zeta(3)-\frac{\pi^{4}}{48}-\frac{\log ^{2} 2}{12} \pi^{2}+\frac{\log ^{4} 2}{12} .
$$

It would be very interesting to find a more general context for these formulas which also have applications to Mahler measure [18.

Finally, one could continue to explore the consequences of Theorem 3 for roots of unity and further implications in other areas such as Feynman diagrams and Mahler measure.

Acknowledgements. We are grateful to Takashi Nakamura and Herbert Gangl for bringing our attention to [22] and to [24] respectively. We are indebted to Karl Dilcher and to Takashi Nakamura for helpful references regarding equation (19). We would like to thank Karl Dilcher, Herbert Gangl, Takashi Nakamura, and Tatiana Hessami Pilehrood for stimulating discussions and the referee for carefully checking the manuscript. 


\section{REFERENCES}

[1] T. M. Apostol. Introduction to analytic number theory. Undergraduate Texts in Mathematics. Springer-Verlag, New York-Heidelberg, 1976. MR 0434929

[2] T. Arakawa, M. Kaneko. On multiple L-values. J. Math. Soc. Japan 56 (2004), no. 4, 967991. MR 2091412

[3] J. M. Borwein, D. M. Bradley, D. J. Broadhurst. Evaluations of $k$-fold Euler/Zagier sums: a compendium of results for arbitrary $k$, Electronic J. Combin. 4 (1997), no. 2, \#R5. MR 1444152

[4] J. M. Borwein, D. Bailey, R. Girgensohn. Experimentation in mathematics: Computational paths to discovery. A K Peters, Natick, MA, 2004. MR 2051473

[5] J. M. Borwein, W. Zudilin. Math Honors: Multiple Zeta Values. CARMA, The University of Newcastle, 2011. http://carma.newcastle.edu.au/MZVs/mzv.pdf

[6] D. W. Boyd. Mahler's measure and special values of $L$-functions. Experiment. Math. 7 (1998), no. 1, 37-82. MR 1618282

[7] D. J. Broadhurst. Massive 3-loop Feynman diagrams reducible to $\mathrm{SC}^{*}$ primitives of algebras of the sixth root of unity. Eur. Phys. J. C Part. Fields 8 (1999), no. 2, 313-333. MR 1739510

[8] F. Brown. Mixed Tate motives over $\mathbb{Z}$. Ann. of Math. (2) 175 (2012), no. 2, 949-976. MR 2993755

[9] C. Deninger. Deligne periods of mixed motives, $K$-theory and the entropy of certain $\mathbb{Z}^{n}$ actions. J. Amer. Math. Soc. 10 (1997), no. 2, 259-281. MR 1415320

[10] L. Euler. Meditationes circa singulare serierum genus. Novi Comm. Acad. Sci. Petropol. 20 (1776), 140-186. http://eulerarchive.maa.org/pages/E477.html

[11] A. B. Goncharov. Polylogarithms in arithmetic and geometry. In: Proceedings of the International Congress of Mathematicians, Vol. 1, 2 (Zürich, 1994), 374-387, Birkhäuser, Basel, 1995. MR 1403938

[12] A. Granville. A decomposition of Riemann's zeta-function. In: Analytic number theory (Kyoto, 1996), 95-101, London Math. Soc. Lecture Note Ser., 247, Cambridge Univ. Press, Cambridge, 1997. MR 1694987

[13] M. E. Hoffman. The algebra of multiple harmonic series. J. Algebra 194 (1997), no. 2, 477495. MR 1467164

[14] M. E. Hoffman, References on multiple zeta values and Euler sums. http://www.usna.edu/ Users/math/meh/biblio.html

[15] M. N. Lalín, Some examples of Mahler measures as multiple polylogarithms, J. Number Theory 103 (2003), no. 1, 85-108. MR 2008068

[16] M. N. Lalín. Mahler measure of some $n$-variable polynomial families, J. Number Theory 116 (2006), no. 1, 102-139. MR 2197862

[17] M. N. Lalín. On certain combination of colored multizeta values, J. Ramanujan Math. Soc. 21 (2006), no. 1, 115-127. MR 2226356

[18] M. N. Lalín. A new method for obtaining polylogarithmic Mahler measure formulas. Res. Number Theory 2 (2016), Art. 17, 16 pp. MR 3543241

[19] M. N. Lalín, J.-S. Lechasseur. Higher Mahler measure of an $n$-variable family, Acta Arith. 174 (2016), no. 1, 1-30. MR 3517530

[20] T. Nakamura. Double Lerch series and their functional relations. Aequationes Math. $\mathbf{7 5}$ (2008), no. 3, 251-259. MR 2424133

[21] T. Nakamura. Double Lerch value relations and functional relations for Witten zeta functions. Tokyo J. Math. 31 (2008), no. 2, 551-574. MR 2477890

[22] T. Nakamura. A simple proof of the functional relation for the Lerch type Tornheim double zeta function, Tokyo J. Math. 35 (2012), no. 2, 333-337. MR 3058710

[23] N. Nielsen. Traité élémentaire des nombres de Bernoulli. Gauthier-Villars, Paris, 1923.

[24] E. Panzer, The parity theorem for multiple polylogarithms, J. Number Theory 172 (2017), 93-113. MR 3573145 
[25] F. Rodriguez-Villegas. Modular Mahler measures. I. In: Topics in number theory (University Park, PA, 1997), 17-48, Math. Appl., 467, Kluwer Acad. Publ., Dordrecht, 1999. MR 1691309

[26] D. Zagier. Evaluation of the multiple zeta values $\zeta(2, \ldots, 2,3,2, \ldots, 2)$. Ann. of Math. (2) 175 (2012), no. 2, 977-1000. MR 2993756

M. N. Lalín $\bowtie$

Département de mathématiques et de statistique

Université de Montréal

CP 6128, succ. Centre-ville

Montréal, QC H3C 3J7, Canada

mlalin@dms . umontreal.ca

J.-S. Lechasseur

Département de mathématiques

Collège André-Grasset

1001 Boul Crémazie E

Montréal, QC H2M 1M3, Canada

jeanseb1777@gmail.com

Received: May 19, 2017

Accepted: February 13, 2018 